\title{
X-ray Reflectivity and Polarized Neutron Reflectivity Investigations of $\left[\mathrm{Co}_{60} \mathrm{Fe}_{60} \mathrm{~B}_{20} / \mathrm{MgO}\right]_{n}$ Multilayers
}

\author{
M. VAdAlì $\grave{a}^{a}, \mathrm{M} \mathrm{WolfF}^{a}, \mathrm{~K} . \mathrm{Westerholt}^{a}, \mathrm{H} . \mathrm{ZABel}^{a}$, \\ P. Wisniowski ${ }^{b}$ AND S. CARDoso ${ }^{b}$ \\ ${ }^{a}$ Experimentalphysik/Festkörperphysik, Ruhr-Universität Bochum \\ Universitätsstraße 150, 44780 Bochum, Germany \\ ${ }^{b}$ INESC-Microsystems and Nanotechnologies \\ Rua Alves Redol 9, 1000- 029 Lisboa, Portugal
}

\begin{abstract}
In recent years magnetic tunnel junctions have been intensively studied and incorporated in magnetic random access memory devices and magnetic sensors, where large tunnelling magnetoresistance ratios are preferred. The largest tunnelling magnetoresistance values until now have been observed in magnetic tunnel junctions with $\mathrm{MgO}$ barriers and $\mathrm{CoFeB}$ electrodes after annealing of the junction above the recrystallization temperature of the amorphous $\mathrm{CoFeB}$ layers. We used X-ray reflectivity and polarized neutron reflectivity to study $\left[\mathrm{Co}_{60} \mathrm{Fe}_{60} \mathrm{~B}_{20} / \mathrm{MgO}\right]_{\mathrm{x} 14}$ multilayers with the $\mathrm{MgO}$ layers prepared by different methods and annealed at different temperatures in order to compare the interface quality and the structural changes induced upon annealing.
\end{abstract}

PACS numbers: 75.50.-i, 75.50.Kj, 75.50.Cc

\section{Introduction}

Magnetic tunnel junctions (MTJs) consisting of two ferromagnetic electrode layers separated by a thin tunnelling barrier and exhibiting a large magnetoresistance at room temperature $[1,2]$ are of great technical importance and are nowadays routinely incorporated in magnetic random access memory (MRAM) devices and magnetic sensors. An actual overview on the development of MTJ appeared in [3]. For technological applications large tunneling magnetoresistance (TMR) ratios and resistance area (RA) product in the range of $1-1000 \Omega \mathrm{m}^{2}$ are needed. Until recently most work has been devoted to junctions with $\mathrm{AlO}_{x}$ barriers, where TMR values up to $70 \%$ have been observed [4]. Large TMR ratios 
require an excellent quality of the interfaces and a small nonconformal roughness [5], which is known to degrade the $\mathrm{TMR}^{\mathrm{AlO}} \mathrm{Al}_{x}$ based tunnel junctions with polycrystalline ferromagnetic metals as electrodes have conventionally been used in MTJs. Amorphous ferromagnetic electrodes, mainly consisting of alloys of $3 d$ ferromagnets combined with $\mathrm{Si}$ or $\mathrm{B}$, typically $\mathrm{CoFeB}$, have also been used and were reported to have very smooth interfaces with values down to $0.4 \mathrm{~nm}$ for the interface roughness [6].

Recently TMR values, as high as $230 \%$ at room temperature, have been reported by different groups for $\mathrm{MgO}$ based MTJs [7, 8], and now the international focus in MTJ research is on $\mathrm{CoFeB} / \mathrm{MgO}$ junctions. In their amorphous state these junctions have the advantage to be homogeneous at small scales, as required for high density recording media. For achieving a large TMR value these junctions have to be annealed at temperatures between $250^{\circ} \mathrm{C}$ and $360^{\circ} \mathrm{C}$. Annealing at $250^{\circ} \mathrm{C}$ causes a partial crystallization of $\mathrm{CoFeB}$, whereas annealing at higher temperatures $\left(360^{\circ} \mathrm{C}\right.$ to $\left.400^{\circ} \mathrm{C}\right)$ leads to a full $\mathrm{CoFeB}$ recrystallization.

The annealing effects on the microscopic structure of these MTJs are presently not completely understood. The restrictions in forming an ideally flat $\mathrm{CoFeB} / \mathrm{MgO}$ interface might be ascribed to the growing process as well as to the intermixing, which gives rise to compositional grading. Recent results obtained with electron microscope pictures [9] suggested that the high TMR ratio is related to the electrode/barrier interface and the developing crystallization of the $\mathrm{CoFeB}$ on annealing at $265^{\circ} \mathrm{C}$.

Here we present a structural study of $\left[\mathrm{Co}_{60} \mathrm{Fe}_{20} \mathrm{~B}_{20} / \mathrm{MgO}\right]$ multilayers, grown on a $\mathrm{Si} / \mathrm{SiO}_{2}$ substrate using the three different processes for the preparation of the $\mathrm{MgO}$ layers, which are commonly also applied in the literature for the preparation of the barrier. This is natural oxidation of $\mathrm{Mg}$, plasma oxidation of $\mathrm{Mg}$, and direct sputtering of $\mathrm{MgO}$. We also observe the changes of the structural quality of the multilayers after annealing at different temperatures. X-ray reflectivity is used to characterize multilayer periods, thickness fluctuations, and the interface roughness, whereas polarized neutron reflectivity (PNR) provides information on the magnetic layer properties.

\section{Preparation and experimental}

The $[\mathrm{CoFeB} / \mathrm{MgO}]$ multilayers were grown on a $\mathrm{Si} / \mathrm{SiO}_{2}$ substrate with the layer sequence given in Table I. They were prepared at INESC by ion beam deposition in a Nordiko36000 system [10], with a base pressure of $2 \times 10^{-9}$ mbar. Three different methods were used to prepare the $\mathrm{MgO}$ layers. In the first series the $\mathrm{MgO}$ layer was obtained by oxidizing $\mathrm{Mg}$ in a natural flow of oxygen, $20 \mathrm{sccm} \mathrm{O}_{2}$ flux for $120 \mathrm{~s}$; in the second series $\mathrm{Mg}$ was oxidized in an oxygen plasma environment in $2 \mathrm{sccm} \mathrm{O}_{2}$ flux with $\mathrm{Ar}$ as inert gas for $45 \mathrm{~s}$, and the third series was prepared by direct reactive sputtering from an $\mathrm{MgO}$ target, using $4 \mathrm{sccm}$ Xe gas for $100 \mathrm{~s}$.

After deposition and in order to check the thermal stability, the samples were annealed at different temperatures, up to $360^{\circ} \mathrm{C}$. 
Multilayer design (nominal thicknesses expressed in $\mathrm{nm}$ ) and $\mathrm{Mg}$ oxidation process of the samples investigated.

\begin{tabular}{l|l|l}
\hline \hline Sample ID & \multicolumn{1}{|c|}{ Multilayer structure $(\mathrm{nm})$} & Mg oxidation conditions \\
\hline 1NAT & $\begin{array}{l}{\left[\mathrm{Co}_{60} \mathrm{Fe}_{20} \mathrm{~B}_{20}(3) / \mathrm{Mg}(1.6)+\mathrm{Ox} .\right]_{\mathrm{x} 14} /} \\
\mathrm{Co}_{60} \mathrm{Fe}_{20} \mathrm{~B}_{20}(3) / \mathrm{MgO}(3)\end{array}$ & Natural oxidation \\
\hline 2REMOT & $\begin{array}{l}{\left[\mathrm{Co}_{60} \mathrm{Fe}_{20} \mathrm{~B}_{20}(3) / \mathrm{Mg}(1.6)+\mathrm{Ox} .\right]_{\mathrm{x} 14} /} \\
\mathrm{Co}_{60} \mathrm{Fe}_{20} \mathrm{~B}_{20}(3) / \mathrm{MgO}(3)\end{array}$ & $\begin{array}{l}\text { Remote plasma } \\
\text { oxidation }\end{array}$ \\
\hline 3REACT & $\begin{array}{l}\left.\mathrm{Co}_{60} \mathrm{Fe}_{20} \mathrm{~B}_{20}(3) / \mathrm{MgO}(1.2)\right]_{\mathrm{x} 14} / \\
\mathrm{Co}_{60} \mathrm{Fe}_{20} \mathrm{~B}_{20}(3) / \mathrm{MgO}(3)\end{array}$ & $\begin{array}{l}\text { Reactive } \mathrm{MgO} \\
\text { deposition }\end{array}$ \\
\hline
\end{tabular}

The X-ray scattering reflectivity (XRR) measurements were performed at the beamline 9 of the DELTA Synchrotron source at the University of Dortmund [11]. We used an X-ray wavelength of $1.1272 \AA$ which corresponds to an energy of $11 \mathrm{keV}$. A pair of crossed slits defined a beam size at the sample position of $1.5 \times 2 \mathrm{~mm}^{2}$, with a detector slit of $0.4 \mathrm{~mm}$ width. Polarized neutron reflectivity measurements [12-14], which allow us to investigate the magnetization profile and to follow the development of the spin structure, were carried out at the Institute Laue Langevin using the ADAM reflectometer $[15,16]$. The experimental setup is such that the polarization axis and the applied field direction are parallel to the sample surface and perpendicular to the scattering plane. The instrument is operating with cold neutrons of wavelength $0.441 \mathrm{~nm}$ which can be optionally polarized and analyzed by transmission supermirrors and spin flippers in front or behind the sample. This provides four cross-sections $R^{++}, R^{--}, R^{+-}$, and $R^{-+}$. Here $(+)$refers to spin-up polarization, $(-)$to spin-down polarization. In the following we will consider only the non-spin flip reflectivities: $R^{++}$and $R^{--}$.

\section{Results}

\subsection{X-rays reflectivity}

In Figs. 1 and 2 we compare the X-ray reflectivity (XRR) for samples 1NAT and 2REMOT in the as-deposited state and the state after thermal annealing at $330^{\circ} \mathrm{C}$ and $360^{\circ} \mathrm{C}$.

We observe Kiessig fringes at least up to the first order Bragg peak and in some cases even beyond. Furthermore, multilayer Bragg peaks can be resolved up to the second order for the remotely oxidized multilayers and up to the third order for the naturally oxidized samples, indicating that the interfaces are relatively flat. One notices that after the annealing there are only very slight changes in the XRR curves, thus at the first glance the multilayer structure is only weakly influenced by the annealing process.

For a quantitative analysis the XRR data were simulated in order to retrieve quantitative information on the thickness and interface roughness. We used the WinGXA code which applies a genetic algorithm to adapt the model structure to 

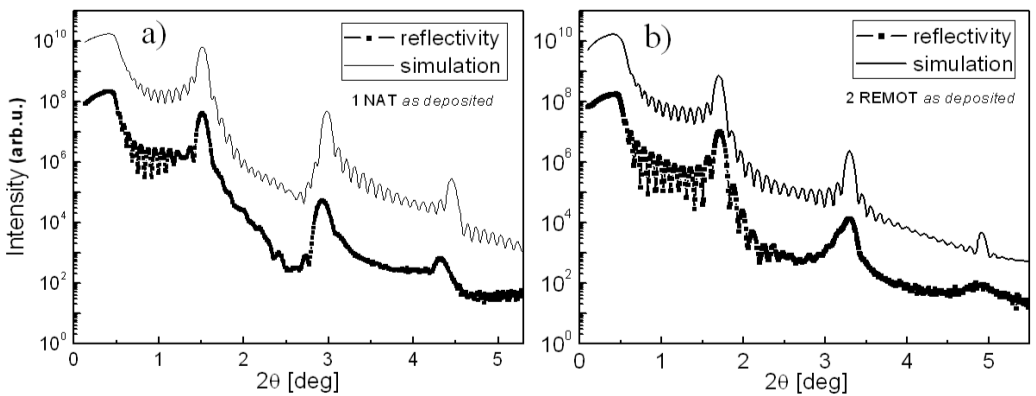

Fig.

1. X-ray

reflectivity

curves for

the

multilayers

$\left[\mathrm{Co}_{60} \mathrm{Fe}_{20} \mathrm{~B}_{20}(3 \mathrm{~nm}) / \mathrm{MgO}(1.6 \mathrm{~nm})\right]_{\mathrm{x} 14}$ 1NAT (a) and 2REMOT (b) in the as-deposited state. The solid lines are simulations. The experimental curves are off-set from the simulation for clarity.
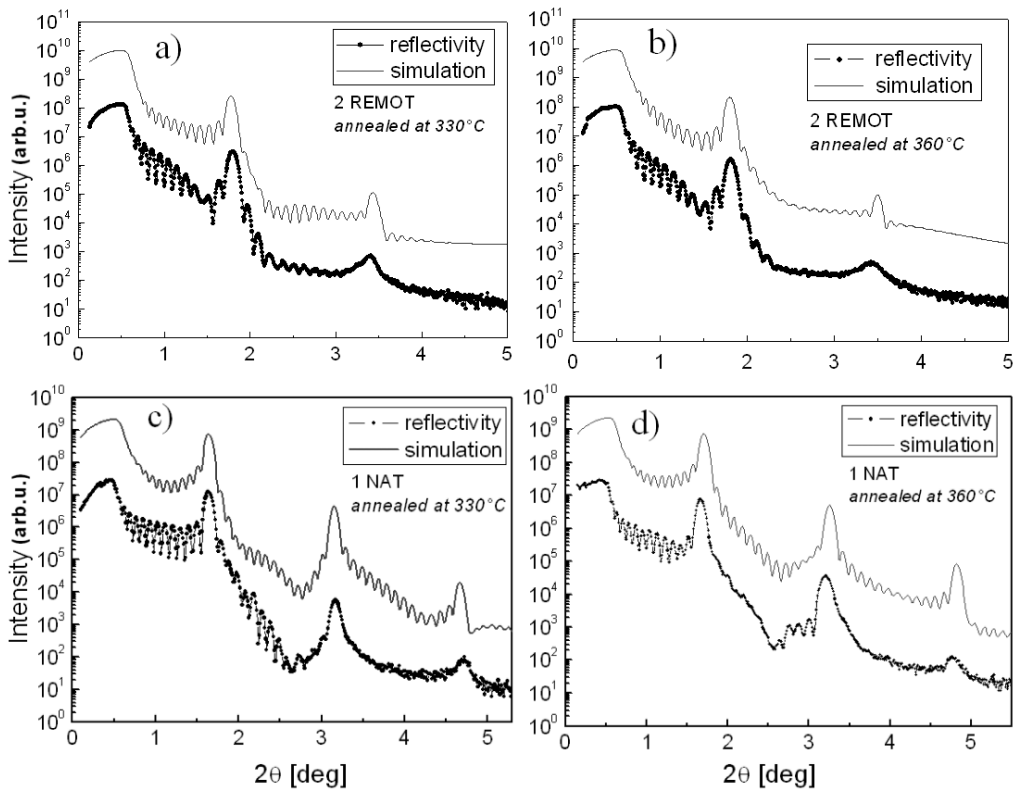

Fig. 2. X-ray reflectivity curves for the multilayers $\left[\mathrm{Co}_{60} \mathrm{Fe}_{20} \mathrm{~B}_{20}(3 \mathrm{~nm}) / \mathrm{MgO}(1.6 \mathrm{~nm})\right]_{\mathrm{x} 14}$ 2REMOT annealed at $330^{\circ} \mathrm{C}$ (a) and annealed at $360^{\circ} \mathrm{C}(\mathrm{b})$ and $1 \mathrm{NAT}$ annealed at $330^{\circ} \mathrm{C}$ (c) and annealed at $360^{\circ} \mathrm{C}(\mathrm{d})$. The solid lines are simulations. The experimental curves are off-set from the simulation for clarity.

the experimental data by an iterative process. [17]. The experimental parameters resulting from the fit are listed in Table II. The interface roughness of the $\mathrm{MgO}$ layers is definitely smaller for the multilayer prepared by natural oxidation than for the plasma oxidation. Moreover, for the natural oxidation the annealing 
hardly affects the roughness parameter, whereas for the plasma oxidized samples the roughness definitely increases upon annealing. This indicates that the interdiffusion at the interfaces upon annealing is larger for the case of plasma oxidation. This type of interdiffusion might destroy the insulating property of the barrier and must be avoided in the preparation process of MTJs. Thus our X-ray reflectivity results indicate that the natural oxidation should be the superior technique for preparing high quality $\mathrm{MgO}$ barriers in MTJs.

TABLE II

Structural parameters of the multilayers with the experimental parameters obtained from X-ray reflectivity fit.

\begin{tabular}{l|c|c|c|c}
\hline \hline \multirow{2}{*}{ Sample ID } & \multicolumn{2}{|c|}{ Bilayer thickness $(\mathrm{nm})$} & \multicolumn{2}{c}{ Roughness $(\mathrm{nm})$} \\
\cline { 2 - 5 } & nominal $( \pm 0.1)$ & fit $( \pm 0.1)$ & $\mathrm{CoFeB}( \pm 0.1)$ & $\mathrm{MgO}( \pm 0.1)$ \\
\hline $\begin{array}{l}\text { 1NAT } \\
\text { as-deposited }\end{array}$ & 4.6 & 4.28 & 0.5 & 0.6 \\
\hline $\begin{array}{l}\text { 1NAT } \\
\text { anneal. } 330^{\circ} \mathrm{C}\end{array}$ & 4.6 & 4.15 & 0.5 & 0.5 \\
\hline $\begin{array}{l}1 \mathrm{NAT} \\
\text { anneal. } 360^{\circ} \mathrm{C}\end{array}$ & 4.6 & 4.05 & 0.4 & 0.5 \\
\hline $\begin{array}{l}\text { 2REMOT } \\
\text { as-deposited }\end{array}$ & 4.6 & 3.78 & 0.6 & 1.4 \\
\hline $\begin{array}{l}\text { 2REMOT } \\
\text { anneal. 330 }\end{array}$ & 4.6 & 3.8 & 0.8 & 1.8 \\
\hline $\begin{array}{l}\text { 2REMOT } \\
\text { anneal. } 360^{\circ} \mathrm{C}\end{array}$ & 4.6 & 3.7 & 0.7 & 1.9
\end{tabular}

Regarding the bilayer thicknesses determined from the X-ray reflectivity in Table II one sees that it definitely shrinks upon annealing. Especially for the naturally oxidized sample this effect is quite large, the bilayer thickness decreases by about $5 \%$ after annealing at $360^{\circ} \mathrm{C}$. This shrinking of the bilayer period can be attributed to the increase in the density of the CoFeB layers in the crystalline state, which is typically of the order of $1 \%$. However, since the decrease in the bilayer period we observe is much larger, we think that the main effect originates from rearrangements of atoms at the interfaces.

If you look carefully at Fig. $2 \mathrm{~d}$, you may notice a detail which the simulations cannot reproduce, namely at broadening of the third Bragg peak, which shows up for the samples 1NAT (as-deposited and annealed). This feature may be attributed to diffuse scattering from roughness correlations; detailed simulations and data analysis about are in progress.

In order to determine the in-plane roughness correlations, transverse scans were taken across the first order Bragg peaks of all respective multilayers. For all 
multilayers the transverse scans (not shown here) are very similar, consisting of a sharp specular component and a broad diffuse component. From the FWHM of the diffuse component we estimate an average in-plane roughness correlation length of about $1-1.2 \mathrm{~nm}$.

\subsection{Polarized neutron reflectivity}

Polarized neutron reflectivity measurements were carried out on three samples, each belonging to a different series (see Table I) and all annealed at $T=280^{\circ} \mathrm{C}$. The measurements were taken at room temperature and in magnetic saturation of the $\mathrm{CoFeB}$ films by applying a magnetic field of $1 \mathrm{kOe}$ parallel to the neutron polarization axis and normal to the scattering plane.

The reflectivity curves for spin-up neutrons $R^{++}$and spin down neutrons $R^{--}$for the three samples are plotted in Fig. 3. The splitting between $R^{++}$and

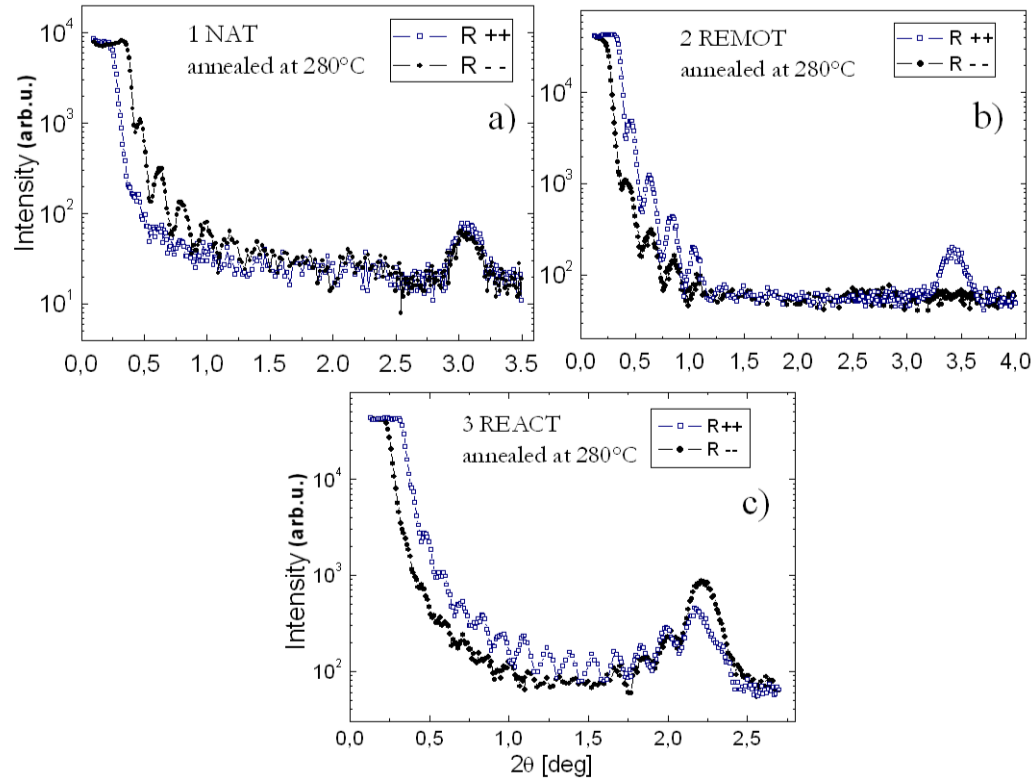

Fig. 3. Polarized neutron reflectivity curves $R^{++}$and $R^{--}$for three multilayers annealed at $280^{\circ} \mathrm{C}$ with $\mathrm{MgO}$ deposited at different conditions (see Table I).

$R^{--}$caused by the magnetic scattering of the ferromagnetic CoFeB layers clearly shows up for all three samples at the total reflection edge and in the total thickness oscillations. At the first multilayer Bragg peak, however, we observe an interesting feature: in Fig. 3a, i.e. for the sample with natural oxidation, the splitting between $R^{++}$and $R^{--}$vanishes at the first Bragg peak, in Fig. 3b, i.e. for the plasma oxidized $\mathrm{MgO}$ films, there is no observable first order Bragg peak for the $R^{--}$ channel and for Fig. 3c, i.e. for the directly sputtered $\mathrm{MgO}$ layers there is a Bragg peak for both channels with a definite splitting with the $R^{--}$intensity larger than 
the $R^{++}$intensity. We reproduce this interesting spin asymmetry in Fig. 4 once more.

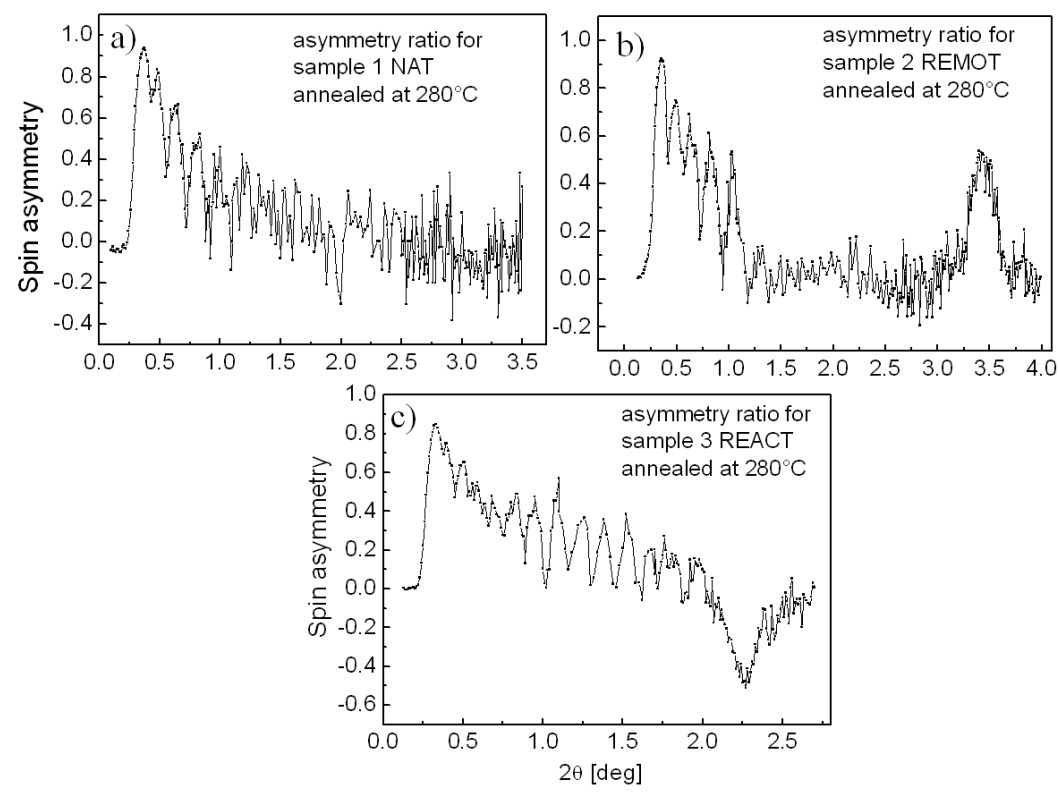

Fig. 4. Spin asymmetry $\left(R^{++}-R^{--}\right) /\left(R^{++}+R^{--}\right)$for the samples of Fig. 3 .

The reason for this feature is that for stoichiometric $\mathrm{MgO}$ and for $\mathrm{Co}_{60} \mathrm{Fe}_{20} \mathrm{~B}_{20}$ the nuclear scattering length densities coincide accidentally $\left(\mathrm{N}_{\mathrm{b}}(\mathrm{MgO})=\mathrm{N}_{\mathrm{b}}(\mathrm{CoFeB})\right)$. For this special case the multilayer Bragg peaks vanish for unpolarized neutrons. For polarized neutrons the intensity of the Bragg peaks is solely due to magnetic scattering and the $R^{--}$and the $R^{++}$channels have the same intensity. Correspondingly, if $R^{++}>R^{--}$at the Bragg peak, MgO has a lower nuclear density than CoFeB, which is the case for sample 2REMOT. Vice versa, if $R^{++}<R^{--}$, the $\mathrm{MgO}$ density is higher than the CoFeB density, which is the case for sample $3 \mathrm{REACT}$.

Thus we may conclude that the scattering length densities for the $\mathrm{MgO}$ layers are different for the three preparation methods. This argument is based on the reasonable assumption that the density of $\mathrm{CoFeB}$ is the same for all three samples. While with PNR measurements we clearly observe a density variation within the $\mathrm{MgO}$ layer, we cannot tell whether the density variation in $\mathrm{MgO}$ is due to $\mathrm{Mg}$ or to O. However, it seems plausible that a smaller density is connected with some oxygen deficiency and vacancies on the O-sublattice, whereas a larger density is connected with excess oxygen and some oxygen atoms on interstitial positions. Thus our results suggest that only the naturally oxidized $\mathrm{MgO}$ layers acquire the proper $\mathrm{MgO}$ density and the correct stoichiometry. 
We also analyzed the off-specular scattering range but did not observe any sizable diffuse scattering intensity, meaning that the magnetic domains are larger than the neutron coherence length. This is expected for a magnetically saturated sample with a negligible interface magnetic roughness.

\section{Conclusions}

Our investigations of $\left[\mathrm{Co}_{60} \mathrm{Fe}_{60} \mathrm{~B}_{20} / \mathrm{MgO}\right]_{\mathrm{x} 14}$ multilayers by X-ray and polarized neutron reflectivity revealed that the interfaces between $\mathrm{CoFeB}$ and $\mathrm{MgO}$ are smooth and do not deteriorate markedly when the sample is annealed at high temperatures. Some additional interdiffusion at high annealing temperatures cannot be excluded, however, especially for the samples with the MgO layers prepared by plasma oxidation. From the neutron reflectivity data we infer that the nuclear density of the $\mathrm{MgO}$ layers in the $\mathrm{CoFeB} / \mathrm{MgO}$ multilayers definitely depends on the preparation method. Our results suggest that only the naturally oxidized $\mathrm{MgO}$ layers have the correct stoichiometry, for the plasma oxidation there is some oxygen deficiency, for the reactive sputtering of $\mathrm{MgO}$ there is an oxygen excess. The problem of an off stoichiometry in reactive sputtering is known in the production of MTJs and must be controlled by choosing the right mixture of $\mathrm{Ar}$ and $\mathrm{O}_{2}$ as sputter gas. Our samples provide evidence that natural oxidation of $\mathrm{Mg}$ at least from a structural point of view gives the highest quality layers. Using the other two standard methods for the barrier preparation, i.e. plasma oxidation or direct $\mathrm{MgO}$ sputtering, one must carefully control the oxygen stoichiometry, which in practice is not easy.

\section{Acknowledgments}

The authors gratefully acknowledge C. Sternemann, M. Paulus, G. Nowak, and A. Remhof for their kind support and assistance during the experiments at the synchrotron source Delta, Dortmund. The financial support provided through the European Unions Marie Curie actions (Research Training Networks) ULTRASMOOTH under contract MRTN-CT-2003-504462 is also gently acknowledged.

\section{References}

[1] J.S. Moodera, L.R. Kinder, T.M. Wong, R. Meservey, Phys. Rev. Lett. 74, 3273 (1995)

[2] T. Miyazaki, N. Tezuka, J. Magn. Magn. Mater. 139, L231 (1995).

[3] G. Reiss, J. Schmalhorst, A. Thomas, A. Hütten, S. Yuasa, in: Magnetic Heterostructures, Advances and Perspectives in Spinstructures and Spintransport, Eds. H. Zabel, S.D. Bader, in series: Springer Tracts in Modern Physics, Vol. 227, p. 282.

[4] D.X. Wang, C. Nordman, J.M. Daughton, Z.H. Qian, J. Fink, IEEE Trans. Magn. 40, 2269 (2004).

[5] A.T.G Pym, A. Lamperti, B.K. Tanner, T. Dimopoulos, M. Rührig, J. Wecker, Appl. Phys. Lett. 88, 162505 (2006). 
[6] A. Kaüfler, Y. Luo, K. Samwer, G. Gieres, M. Vieth, J. Wecker, J. Appl. Phys. 91, 1701 (2002).

[7] D.D. Djayaprawira, K. Tsunekawa, M. Nagai, H. Maehara, S. Yamagata, N. Watanabe, S. Yuasa, Y. Suzuki, K. Ando, Appl. Phys. Lett. 86, 092502 (2005).

[8] S.S.P. Parkin, C. Kaiser, A. Panchula, P.M. Rice, B. Hughes, M. Samant, S.-H. Yang, Nat. Mater. 3, 862 (2004).

[9] F.F. Li, R. Sharif, L.X. Jiang, X.Q. Zhang, X.F. Han, Y. Wang, Z. Zhang, J. Appl. Phys. 98, 113710 (2005).

[10] Z.G. Zhang, P.P. Freitas, A.R. Ramos, N.P. Barradas, J.C. Soares, J. Appl. Phys. 91, 8786 (2002).

[11] C. Krywka, M. Paulus, C. Sternemann, M. Volmer, A. Remhof, G. Nowak, A. Nefedov, B. Pöter, M. Spiegel, M. Tolan, J. Synchr. Rad. 13, 8 (2006).

[12] H. Zabel, Physica B 198, 156 (1994).

[13] R.P. Felcher, R.O. Hilleke, R.K. Crawford, J. Haumann, R. Kleb, G. Ostrowski, Rev. Sci. Instrum. 58, 609 (1987).

[14] C.F. Majkrzak, Physica B 619, 156 (1989).

[15] A. Schreyer, R. Siebrecht, U. Englisch, U. Pietsch, H. Zabel, Physica B 248, 349 (1998).

[16] M. Wolff, K. Zhernenkov, H. Zabel, Thin Solid Films 515, 5712 (2007).

[17] A.J.G. Leenaers, D.K.G. de Boer, X-Ray Spect. 26, 115 (1997). 